\title{
A conservative theory of gradable modality *
}

\author{
Robert Pasternak \\ Stony Brook University
}

\begin{abstract}
In the tradition of modal semantics stemming from the work of Kratzer (1981, 1991), several attempts have been made to analyze adjectives like important, which are both modal and gradable. I show that existing theories of such gradable modal adjectives (GMAs) in this framework do not allow for comparisons across distinct sets or prioritizations of premises, as the basis for comparison in these theories is too closely wedded to the particular choice and ranking of priorities. To fix this, I include modal degrees in the ontology, with sets of premises being relativized to those degrees. What results is a theory that allows comparison across sets of priorities, while still retaining the premise-based world ordering and quantification that lies at the heart of Kratzer's approach. This theory is then extended to account for existentially-quantifying GMAs like permissible, as well as entailment relations between modal auxiliaries and GMAs.
\end{abstract}

Keywords: modality, gradability, possible world semantics, adjectives, cross-polarity

\section{Introduction}

The adjective important lies at the intersection of two productive research programs in semantics: gradability and modality. That important is gradable can be seen in (1): (1a) shows that it is compatible with a variety of degree modifiers, and (1b) that it can appear in comparative constructions. The modal interpretation of important is established in (2), which illustrates that, given a fixed context, must $p$ non-mutually entails it is important that $p$, which in turn non-mutually entails should $p$. Hence, $(2 \mathrm{a}, \mathrm{c})$ are acceptable, while $(2 \mathrm{~b}, \mathrm{~d})$ are judged to be contradictory.

(1) a. It's somewhat/very/extremely important that you do the dishes.

b. It's more important that you do the dishes than it is that you walk the dog.

(2) a. It's important that you do the dishes, but it's not the case that you must.

* For their insightful comments and critiques, I am grateful to Thomas Graf, Richard Larson, Paul Portner, and Ildikó Emese Szabó. Thanks also to audiences at Stony Brook University, the NYU Semantics Group, the Workshop on Modality Across Categories (Universitat Pompeu Fabra), and SALT 26; comments by Peter Klecha, Alda Mari, and Aynat Rubinstein were particularly helpful. 
b. \# You must do the dishes, but it's not important that you do so.

c. You should do the dishes, but it's not important that you do so.

d. \# It's important that you do the dishes, but it's not the case that you should.

Theories of such gradable modal adjectives (GMAs) have generally fallen into three categories. Kratzer (1991, 2012), Villalta (2008), Katz, Portner \& Rubinstein (2012), and Portner \& Rubinstein (2014) adopt what I will call conservative theories of gradable modality, in which all modality - gradable or otherwise - involves premise-based world ordering and quantification along the lines of Kratzer 1981. In contrast, Lassiter (2011a, 2011b) proposes what might be called a liberal theory: while he agrees that modal auxiliaries and GMAs have the same underlying semantics, he eschews Kratzer's approach in favor of probabilities for epistemic modals and the decision-theoretic notion of expected utility for root modals. Finally, Klecha (2014) offers a non-unified theory, in which auxiliaries utilize world ordering and quantification, while GMAs do not, with entailment relations between auxiliaries and GMAs being captured by meaning postulates.

Restricting my investigation to conservative theories in this paper, I show that previous approaches cannot account for cases in which comparisons are made across distinct sets or rankings of priorities, as the proposed means of comparison is too closely wedded to the particular choice of premises. I resolve this issue by treating modal degrees as ontological primitives: both the set of modal degrees and their ordering exist independently of the choice of priorities. This inclusion of degrees in the ontology is by no means novel, and is a common assumption throughout the literature on gradability (see von Stechow 1984, Kennedy 1997, Beck 2011 for extensive discussion). These degrees are then integrated into a Kratzer-style modal semantics. The analysis is subsequently extended to existentially-quantifying GMAs like permissible, and then further revised in order to capture the entailments in (2). The result is a theory that directly incorporates insights from work on the semantics of gradability, while nonetheless remaining fundamentally true to Kratzer's theory of modality.

\section{Other conservative theories and their discontents}

In this section, I discuss previous conservative theories of gradable modality, and the problems they face when comparing across different sets and rankings of priorities. I focus mostly on a somewhat simplified version of the theory adopted by Portner \& Rubinstein (2014), as it is both the easiest theory in which to formulate the problem and the theory most similar to the one set forth in Section 3. Other conservative theories will receive a brief mention at the end of this section, where we will see that they fare no better. 
A conservative theory of gradable modality

\subsection{Formal underpinnings}

As discussed above, conservative theories take as their starting point Kratzer's (1981, 1991) seminal theory of modality. Kratzer makes use of two context-dependent functions from worlds to sets of propositions: the modal base $(f)$ restricts the set of worlds under consideration, while the ordering source $(g)$ induces a preorder of worlds with respect to a contextually determined set of priorities.

Given a world of evaluation $w$ and context $c$, the way that $f^{c}(w)$ restricts the set of worlds is simple: only worlds in which all the propositions in $f^{c}(w)$ hold will be under consideration. Since a proposition is treated as (the characteristic function of) a set of possible worlds, this means that the set of viable worlds is $\bigcap f^{c}(w)$, which I will refer to as $F_{w}^{c}$. As for the world-ordering generated by $g^{c}(w)$, Kratzer follows Lewis (1981) in adopting the definition of $\preccurlyeq_{A}$ in (3), where $A$ is a set of propositions, and $w_{1} \preccurlyeq A w_{2}$ means that $w_{1}$ is at least as ideal as $w_{2}$ with respect to $\preccurlyeq_{A}$ :

$$
w_{1} \preccurlyeq A w_{2} \text { iff }\left\{p \in A \mid p\left(w_{1}\right)\right\} \supseteq\left\{p \in A \mid p\left(w_{2}\right)\right\}
$$

For Kratzer, modal auxiliaries then quantify over the set of $\preccurlyeq g^{c}(w)$-ideal worlds in $F_{w}^{c}$, with must $p$ asserting that $p$ holds in all ideal worlds, and can $p$ asserting that $p$ holds in at least one ideal world: ${ }^{1}$

$$
\begin{aligned}
& \text { If } \operatorname{BEST}\left(\preccurlyeq g^{c}(w), F_{w}^{c}\right)=\left\{w^{\prime} \in F_{w}^{c} \mid \neg \exists w^{\prime \prime} \in F_{w}^{c}\left[w^{\prime \prime} \prec_{g^{c}(w)} w^{\prime}\right]\right\}, \text { then } \\
& \text { a. } \llbracket m u s t p \rrbracket^{c}=\lambda w . \forall w^{\prime} \in \operatorname{BEST}\left(\preccurlyeq g^{c}(w), F_{w}^{c}\right)\left[p\left(w^{\prime}\right)\right] \\
& \text { b. } \llbracket \text { can } p \rrbracket^{c}=\lambda w . \exists w^{\prime} \in \operatorname{BEST}\left(\preccurlyeq_{g^{c}(w)}, F_{w}^{c}\right)\left[p\left(w^{\prime}\right)\right]
\end{aligned}
$$

The definitions in (4) are, of course, categorical: in a given context, must $p$ and can $p$ are either true or false. But as (1) illustrates, there are scalar, non-categorical varieties of modality as well. The challenge facing conservative theories, then, is how to capture these gradable modal interpertations within Kratzer's framework.

\subsection{Portner \& Rubinstein's theory}

Portner \& Rubinstein (2014), hereafter P\&R, start with three intuitively plausible assumptions. First, GMAs like important quantify over worlds in the same manner as necessity modals. Second, priorities are ranked, with some premises taking higher priority than others. Third, the relative importance of two propositions correlates with the relative rank of the premises that necessitate them: if a high-ranking priority dictates that $p$, while it takes the addition of lower-ranked priorities to make $q$ mandatory, then $p$ is more important than $q$.

1 This presupposes what Lewis (1973) calls the limit assumption: that there is always a non-empty set of maximally ideal worlds. Kratzer (1981) avoids this assumption, but I adopt it for simplicity's sake. 
$\mathrm{P} \& \mathrm{R}$ introduce ranked prioritization by adding a contextually determined function, which I will call $\gamma$, that applies to $g^{c}(w)$ (where $w$ is the world of evaluation), simulating the gradual removal of lower-priority propositions by generating a set of subsets of $g^{c}(w)$ representing the stages in this stepwise elimination of premises. ${ }^{2}$ For instance, imagine that Mary is a secret agent for the CIA, and her team has been tasked with a rescue mission to take place on Saturday. There are three hostages to be rescued, with propositions $p, q$, and $r$ being true in worlds where Pauline, Quinn, and Rhonda, respectively, are rescued on Saturday. The CIA has decided that rescuing Pauline is top priority, followed by Quinn, then Rhonda. In this case, $g^{c}(w)=\{p, q, r\}$, while $\gamma^{c}\left(g^{c}(w)\right)$ contains $\{p, q, r\},\{p, q\}$, and $\{p\}$, which I will call $A, B$, and $C$, respectively. Notice that since rescuing Rhonda is lowest-priority, $r$ is removed first, followed by $q$, with $p$ being highest-priority.

Each of the sets of propositions in $\gamma^{c}\left(g^{c}(w)\right)$ can be used to generate its own world-ordering as in (3). Using the world-orderings $\preccurlyeq_{A}, \preccurlyeq_{B}$, and $\preccurlyeq_{C}, \mathrm{P} \& \mathrm{R}$ construct a scale - an ordered pair consisting of a set of degrees and an ordering of those degrees - as follows. First, for each world-ordering, generate the set of propositions that hold in all ideal worlds in $F_{w}^{c}$ with respect to that ordering: $\left\{s \mid \forall w^{\prime} \in \operatorname{BEST}\left(\preccurlyeq_{A}, F_{w}^{c}\right)\left[s\left(w^{\prime}\right)\right]\right\},\left\{s \mid \forall w^{\prime} \in \operatorname{BEST}\left(\preccurlyeq_{B}, F_{w}^{c}\right)\left[s\left(w^{\prime}\right)\right]\right\}$, and $\left\{s \mid \forall w^{\prime} \in\right.$ $\left.\operatorname{BEST}\left(\preccurlyeq c, F_{w}^{c}\right)\left[s\left(w^{\prime}\right)\right]\right\}$. For this example, I will adopt the simplifying assumption that $p, q, r$, and $F_{w}^{c}$ are all logically independent, i.e., that Mary's team is capable of rescuing any combination of the three hostages. This allows for a simplification of the descriptions of these three sets to $\left\{s \mid p \cap q \cap r \cap F_{w}^{c} \subseteq s\right\},\left\{s \mid p \cap q \cap F_{w}^{c} \subseteq s\right\}$, and $\left\{s \mid p \cap F_{w}^{c} \subseteq s\right\}$. These sets of propositions are the degrees: call them $d_{A}, d_{B}$, and $d_{C}$, with $D_{\alpha}=\left\{d_{A}, d_{B}, d_{C}\right\}$. These degrees are then ordered by the superset relation: $d_{A} \supset d_{B} \supset d_{C}$, so $d_{A}<_{\alpha} d_{B}<_{\alpha} d_{C}$.

With the modal scale $\left\langle D_{\alpha}, \leq_{\alpha}\right\rangle$ now constructed, the denotation of a GMA like important utilizes a measure function taking a proposition and world of evaluation as input, and returning a degree. More specifically, since modal degrees are sets of propositions, the degree returned is the highest degree of which that proposition is a member. In this example, any proposition $p^{\prime}$ entailed by $p \cap F_{w}^{c}$ will have an importance of $d_{C}$ because $p^{\prime} \in d_{C}$. Put in terms of the CIA example, if $p^{\prime}$ must be true in order for Mary's team to rescue Pauline, then $p^{\prime}$ is important to degree $d_{C}$. For some proposition $q^{\prime}$ entailed by $q \cap F_{w}^{c}$ and not by $p \cap F_{w}^{c}$ (i.e., $q^{\prime}$ is necessary only with regards to saving Quinn), the importance of $q^{\prime}$ will be $d_{B}$, as $q^{\prime} \in d_{B}$ and $q^{\prime} \notin d_{C}$. Since $d_{B}<_{\alpha} d_{C}, q^{\prime}$ is less important than $p^{\prime}$. Hence, this theory meets the three intuitions discussed previously: important involves universal quantification

2 P\&R actually follow von Fintel \& Iatridou (2008) in adopting an analysis with multiple, ranked ordering sources, in order to capture distinctions between strong necessity modals like must and weak necessity modals like should. I will return to this idea later on, but it is worth noting that including it here would not resolve the problems faced by P\&R's theory. 
over ideal worlds in the construction of degrees (but see Section 3.4), priorities are ranked by $\gamma$, and the importance of a proposition is contingent on how high the priorities are that demand it.

\subsection{Comparison across ordering sources and prioritizations}

As we have seen, for $\mathrm{P} \& \mathrm{R}$, modal scales are directly derived from prioritized sets of premises. As a result, shifting the priorities around means altering the scale itself. For example, let's keep our priorities as $\{p, q, r\}$, but switch the rankings of $p$ and $r$, so that $\gamma$, when applied to this set, returns $\{\{p, q, r\},\{q, r\},\{r\}\}$. Retaining previous assumptions, this means that the set $D_{\beta}$ of degrees will contain $\left\{s \mid p \cap q \cap r \cap F_{w}^{c} \subseteq\right.$ $s\},\left\{s \mid q \cap r \cap F_{w}^{c} \subseteq s\right\}$, and $\left\{s \mid r \cap F_{w}^{c} \subseteq s\right\}$. The first degree is simply $d_{A}$ again; the second two I will call $d_{B}^{\prime}$ and $d_{C}^{\prime}$, respectively. This new prioritization has thus led to the construction of a new scale, $\left\langle D_{\beta}, \leq_{\beta}\right\rangle$, where $d_{A}<_{\beta} d_{B}^{\prime}<_{\beta} d_{C}^{\prime}$ (since $\left.d_{A} \supset d_{B}^{\prime} \supset d_{C}^{\prime}\right)$.

Given these two prioritizations, the importance of rescuing Quinn will be $d_{B}$ for the scale $\left\langle D_{\alpha}, \leq_{\alpha}\right\rangle$, and $d_{B}^{\prime}$ for the scale $\left\langle D_{\beta}, \leq_{\beta}\right\rangle$. But what is the relationship between $d_{B}$ and $d_{B}^{\prime}$ ? Since $d_{B}=\left\{s \mid p \cap q \cap F_{w}^{c} \subseteq s\right\}$, and $d_{B}^{\prime}=\left\{s \mid q \cap r \cap F_{w}^{c} \subseteq s\right\}$, we know that $d_{B} \nsubseteq d_{B}^{\prime}$, and $d_{B}^{\prime} \nsubseteq d_{B}$. Therefore, $d_{B}$ and $d_{B}^{\prime}$ are incomparable, and in fact, outside of $d_{A}$ none of the degrees in $D_{\alpha}$ are comparable with any in $D_{\beta}$.

In P\&R's theory, then, it is possible to generate two scales with partial overlap in degrees: $d_{A}$ is shared between $D_{\alpha}$ and $D_{\beta}$, but all of the other degrees are incomparable. There are thus several possible predictions for what should happen when trying to make comparisons involving two distinct ordering sources, or two prioritizations of the same ordering source. One possibility is that, in spite of any potential overlap, non-identical modal scales count as fully distinct scales, so that comparison across (prioritizations of) ordering sources should be as ill-formed as other comparisons across different scales, such as (5):

$$
\text { \# Larry is more tired than Michael is clever. (Kennedy 1997: 27) }
$$

A second possibility is that $\left\langle D_{\alpha}, \leq_{\alpha}\right\rangle$ and $\left\langle D_{\beta}, \leq_{\beta}\right\rangle$ are parts of a single partiallyordered scale $\left\langle D_{\omega}, \leq \omega\right\rangle$, where $D_{\omega}=\left\{\left\{s^{\prime} \mid s \subseteq s^{\prime}\right\} \mid s \subseteq W\right\}$, and for all $d, d^{\prime} \in D_{\omega}$, $d \leq{ }_{\omega} d^{\prime}$ iff $d \supseteq d^{\prime}$. In this case, it is possible for comparison across prioritizations to be licit if the circumstances are right and the two degrees happen to be comparable. If the two degrees are not comparable, one predicts either incommensurability effects along the lines of (5), or simple falsehood, depending on one's analysis of incomparable degrees within the same scale.

As it turns out, the evidence points to neither of these predictions being accurate. Consider the following variant of the CIA scenario from before. On Monday, everything was as stated earlier: rescuing Pauline was ranked above rescuing Quinn, 
which was ranked above rescuing Rhonda. However, yesterday (Tuesday), the CIA determined that now, rescuing Rhonda is top priority, followed by Quinn, then Pauline. Once again, assume that nothing prevents Mary's team from successfully rescuing all three. We are therefore looking at a situation in which, on P\&R's theory, the scales of importance on Monday and today are different: on Monday, the scale is $\left\langle D_{\alpha}, \leq_{\alpha}\right\rangle$, and today the scale is $\left\langle D_{\beta}, \leq_{\beta}\right\rangle .{ }^{3}$ As demonstrated above, this means that attempting to compare the relative importance of rescuing Quinn on Monday and today should necessarily lead to deviance or falsehood, regardless of which of the two aforementioned analyses is adopted. However, as an utterance to Mary from her boss, (6) is perfectly well-formed, and potentially true:

(6) It is (currently) as important that you rescue Quinn as it was on Monday.

Comparisons across times are not the only problematic case. In English, important can appear with a prepositional phrase to $N P$, which indicates whose priorities are being considered. Thus, (7) is true iff rescuing Pauline is of sufficiently high importance relative to the priorities of the CIA:

It is important to the CIA that you rescue Pauline.

Putting aside how this is achieved compositionally, a simple analysis of (7) would be that it sets the ordering source to $g^{\mathrm{CIA}}$, the set containing the CIA's priorities. Since the rankings of priorities can vary from individual to individual, on P\&R's theory the presence of this prepositional phrase would presumably also set $\gamma$ to $\gamma^{\mathrm{CIA}}$, which returns the CIA's ranking of their priorities.

With this in mind, let's say that both the CIA and the FBI want Pauline, Quinn, and Rhonda rescued on Saturday, and that they desire nothing else. Therefore, $g^{\mathrm{CIA}}(w)=g^{\mathrm{FBI}}(w)=\{p, q, r\}$. However, the CIA considers saving Pauline to be top priority, followed by Quinn, followed by Rhonda, whereas the FBI has these priorities reversed, with Rhonda ranking above Quinn, who is in turn above Pauline. In other words, $\gamma^{\mathrm{CIA}}\left(g^{\mathrm{CIA}}(w)\right)=\{\{p, q, r\},\{p, q\},\{p\}\}$, while $\gamma^{\mathrm{FBI}}\left(g^{\mathrm{FBI}}(w)\right)=\{\{p, q, r\},\{q, r\},\{r\}\}$. In this case, $\mathrm{P} \& \mathrm{R}$ predict that attempting to compare the importance of saving Quinn to the FBI and to the CIA should lead to deviance or falsehood, for the same reason as for the previous example. However, once again, (8) would be a perfectly acceptable and possibly true statement when said to Mary by her boss:

(8) For you to save Quinn is as important to the FBI as it is to the CIA.

3 Note that I ignore the fact that the set of viable worlds has presumably also changed from Monday to today, since what is circumstantially possible changes over time. This makes things even worse for $\mathrm{P} \& \mathrm{R}$, since for them, changing the set of worlds under consideration also alters the scale. As we will see in Section 3, the theory adopted in this paper faces no such problem. 
Note that (8) becomes no less felicitous if the CIA and FBI have wildly different sets and rankings of priorities, or even priorities that contradict each other's. As a result, the overall picture that arises from examples like (6) and (8) is that the set of modal degrees cannot be entirely parasitic on the choice and ranking of premises, since comparisons can be made across quite different prioritizations.

\subsection{Within-ordering theories and post hoc degree assignment}

While I have only discussed P\&R's theory thus far, things look no better for other conservative approaches. The theories of Kratzer (1991, 2012), Villalta (2008), and Katz et al. (2012) all make modal comparisons based on the relative positions of worlds within a given world-ordering. To concretize this idea a bit, I provide Kratzer's (1991) definition of better possibility in (9); the other theories mentioned above operate on roughly the same principle.

a. Given context $c$ and world of evaluation $w, p$ is at least as good a possibility as $q$ iff for all $q$-worlds $w^{\prime}$ in $F_{w}^{c}$, there exists some $p$-world $w^{\prime \prime}$ in $F_{w}^{c}$ such that $w^{\prime \prime} \preccurlyeq g^{c}(w) w^{\prime}$.

b. Given context $c$ and world of evaluation $w, p$ is a better possibility than $q$ iff $p$ is at least as good a possibility as $q$, but not vice versa.

Presumably, $p$ is more important than $q$ would be analyzed in a similar fashion. But it should be readily apparent that, since these theories based on the comparative positions of worlds can only be defined relative to a given ordering of worlds, they cannot adequately handle comparisons across two distinct world-orderings.

Nonetheless, there is a conceivable solution for all of these conservative theories that warrants some discussion. At its heart, each of these approaches simply serves as a way of ordering propositions by importance. With this in mind, one might propose assigning each proposition in the domain of this propositional ordering to some degree on the ontologically independent modal scale $\langle D, \leq\rangle$, in a manner that preserves this ordering. (That is, $p$ is assigned a higher degree than $q$ iff it is more important than $q$.) Since this scale exists independently of any given ordering source, each ordering source can map onto it, allowing for comparisons across prioritizations.

Unfortunately, such a post hoc assignment of degrees will not work without considerable further stipulation, as there is an infinite number of ways a given ordering of propositions can be transferred onto a densely-ordered scale. In fact, if the domains of the propositional orderings $\leq_{j}$ and $\leq_{k}$ can be mapped to the scale $\langle D, \leq\rangle$, then for any propositions $p$ and $q$ in the domain of $\leq_{j}$ and $\leq_{k}$, respectively, there will always be mappings in which $p$ is assigned a degree higher than-or equal to, or less than-the one $q$ is assigned to, while still maintaining the required 
relationship between each proposition-ordering and the scale. As a result, since the path from premise set to scale is not properly anchored, the relationship between two different mappings onto the same scale is arbitrary, rendering all but meaningless any comparison across those two mappings.

That being said, the basic premise underlying this approach is an appealing one: the problem of comparison across ordering sources can be resolved by properly intertwining world-orderings and ontologically independent degrees. In the next section, I offer a proposal for just how this intertwining between degrees and worldorderings should take place.

\section{The ordering source as degree-relative}

In this section, I propose a means of fusing the Kratzerian tradition of modal semantics with a degree-based semantics of gradability. Before delving into the details of my proposal, however, some discussion of the general architecture of the theory is in order.

\subsection{The theory in broad strokes}

In analyzing the asymmetric entailment from strong necessity modals like must to weak necessity modals like should, von Fintel \& Iatridou (2008) posit that there are two ranked ordering sources, which I will call $g_{1}^{c}$ and $g_{2}^{c}$ (with $g_{1}^{c}$ outranking $\left.g_{2}^{c}\right) .{ }^{4}$ On this approach, must only utilizes the primary ordering source, universally quantifying over the worlds in $\operatorname{BEST}\left(\preccurlyeq g_{1}^{c}(w), F_{w}^{c}\right)$. Meanwhile, should only quantifies over a subset of these worlds: namely, those in $\operatorname{BEST}\left(\preccurlyeq_{g_{2}^{c}(w)}, \operatorname{BEST}\left(\preccurlyeq g_{1}^{c}(w), F_{w}^{c}\right)\right)$. Since universal quantification over a subset is weaker than universal quantification over a superset, must $p$ entails should $p$. On a more intuitive level, must $p$ can be thought of as claiming that $p$ holds in all acceptable worlds, while should $p$ claims that $p$ holds in all ideal worlds, with the inclusion of the lower-ranked premises that distinguish ideal from merely acceptable worlds serving to weaken the modal claim.

As noted above, P\&R's theory operates on what is fundamentally the same principle: the more nitpicky one must be in order to require that $p$ be the case, the less important $p$ is; the less demanding one needs to be to mandate $p$, the more important $p$ is. The theory articulated in this section also falls within this general perspective, but with the added assumption that the modal scale $\langle D, \leq\rangle$ is not derived from the set and/or ranking of priorities, but leads an independent existence separate from the contingencies of any given set or prioritization of premises. Since putting

4 In actuality, von Fintel \& Iatridou (2008) allow for the existence of more than two ordering sources, with a divide occuring between those that are primary and those that are secondary. For the purposes of this paper, this distinction is irrelevant. 
degrees in the ontology is a common move in the semantics of gradability (see von Stechow 1984, Kennedy 1997, Beck 2011 for discussion), I take extending this approach to GMAs to be non-stipulative.

While degrees are separate ontological objects, each degree will be associated with a set of ideal possible worlds, in a manner to be explained shortly. The means of association between degrees and sets of worlds will be such that for any degrees $d_{1}$ and $d_{2}$, where $d_{1}<d_{2}$, the set of worlds associated with $d_{1}$ is a subset of the set of worlds associated with $d_{2}$. This gradual removal of worlds on the way down the scale corresponds to the aforementioned intuition that we are becoming more and more demanding with respect to what constitutes an ideal world. This in turn entails that universally quantifying over the worlds associated with $d_{1}$ will be weaker than universally quantifying over those associated with $d_{2}$, so conversely, universal quantification over associated worlds gets stronger going up the scale.

Following Kennedy $(1997,2007)$ in treating the denotation of an adjective as a measure function, for a given proposition $p$, \important $\rrbracket$ will simply return the highest degree such that $p$ holds in all worlds associated with that degree. That is, if $\operatorname{Assoc}^{c}(w, d)$ is the set of worlds associated with $d$ in context $c$, given a world of evaluation $w$, then important will have a denotation along the lines of (10):

$$
\llbracket \text { important } \rrbracket^{c}=\lambda p \lambda w \cdot \max \left(\left\{d \mid \forall w^{\prime} \in \operatorname{Assoc}^{c}(w, d)\left[p\left(w^{\prime}\right)\right]\right\}\right)
$$

\subsection{Associating degrees and sets of worlds}

The next step is to define how degrees are associated with shrinking sets of possible worlds in the manner just described. A plausible starting place would be to add a degree argument to the ordering source, making it a contextually determined function from worlds and degrees to sets of propositions. The propositions in $g^{c}(w, d)$ (for some $d \in D$ ) can then be thought of as propositions that would be $d$-important were it not for potential conflicting premises and/or prohibitive circumstances, in much the same way that for Kratzer (1981), the propositions in $g^{c}(w)$ are propositions that would be necessary were it not for such possible conflicts.

Naturally, since $g^{c}(w, d)$ is a set of propositions, one can order worlds at aiven degree in the manner described in (3). However, this accomplishes little on its own. There is no guarantee that $\operatorname{BEST}\left(\preccurlyeq g^{c}\left(w, d_{1}\right), F_{w}^{c}\right)$ will be a subset of $\operatorname{BEST}\left(\preccurlyeq g^{c}\left(w, d_{2}\right), F_{w}^{c}\right)$ if $d_{1}<d_{2}$, so this cannot be how degrees are associated with sets of worlds. In other words, the set $\operatorname{BEST}\left(\preccurlyeq_{g^{c}}\left(w, d_{1}\right), F_{w}^{c}\right)$ is not necessarily more demanding than $\operatorname{BEST}\left(\preccurlyeq_{g^{c}\left(w, d_{2}\right)}, F_{w}^{c}\right)$ with regards to what constitutes an ideal set of worlds.

One way of fixing this is to follow the method articulated by von Fintel \& Iatridou (2008). Pretending for the time being that $D$ consists only of the degrees $d_{1}, d_{2}$, and $d_{3}$ (with $d_{1}<d_{2}<d_{3}$ ), we can start by associating with $d_{3}$ the set $\operatorname{BEST}\left(\preccurlyeq_{g^{c}\left(w, d_{3}\right)}, F_{w}^{c}\right)$. Next, the set of worlds associated with $d_{2}$ would be 
$\operatorname{BEST}\left(\preccurlyeq g^{c}\left(w, d_{2}\right), \operatorname{BEST}\left(\preccurlyeq g^{c}\left(w, d_{3}\right), F_{w}^{c}\right)\right)$. Finally, the set of worlds associated with

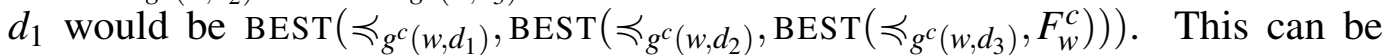
thought of as a "best of the best" approach: going down the scale, a smaller and smaller set of ideal worlds is being considered.

Another possibility is to claim that it is not sets of ideal worlds that trickle down from higher degrees to lower degrees, but world-orderings. Katz et al. (2012) define an operation $*$ that combines two sets of premises $A_{1}$ and $A_{2}$ into a single set of premises, $A_{1} * A_{2}$, which orders worlds as if $A_{2}$ were serving as a tie-breaker for $A_{1}$. That is, if worlds $w_{1}$ and $w_{2}$ are strictly ordered or incomparable with respect to $\preccurlyeq A_{1}$, then $w_{1} \preccurlyeq A_{1} * A_{2} w_{2}$ iff $w_{1} \preccurlyeq A_{1} w_{2}$; but if $w_{1} \approx_{A_{1}} w_{2}$, then $w_{1} \preccurlyeq A_{1} * A_{2} w_{2}$ iff $w_{1} \preccurlyeq A_{2} w_{2}$. As a result, it is guaranteed that $\operatorname{BEST}\left(\preccurlyeq A_{1} * A_{2}, F_{w}^{c}\right)$ is a subset of $\operatorname{BEST}\left(\preccurlyeq A_{1}, F_{w}^{c}\right)$. With this in mind, let $A=g^{c}\left(w, d_{3}\right), B=A * g^{c}\left(w, d_{2}\right)$, and $C=B * g^{c}\left(w, d_{1}\right)$. We can then associate with $d_{3}$ the set of worlds $\operatorname{BEST}\left(\preccurlyeq_{A}, F_{w}^{c}\right)$, with $d_{2}$ the set $\operatorname{BEST}\left(\preccurlyeq_{B}, F_{w}^{c}\right)$, and with $d_{1}, \operatorname{BEST}\left(\preccurlyeq_{C}, F_{w}^{c}\right)$. Once again, we are left with a situation where the set of worlds associated with a lower degree is a subset of the set of worlds associated with a higher degree. This can be considered an approach of increasing discernment: the set of worlds under consideration is the same at each degree level, but at lower degrees, the ordering is more fine-grained.

Both of these aproaches capture in an intuitive manner the notion that on the way down the scale, the list of demands that must be met for worlds to count as ideal grows. But there's a problem. In their current forms, both treatments require that something, whether it be a set of ideal worlds or a world-ordering, be passed from one degree to the next highest degree. However, given the common assumption that scales are densely ordered, there is no such thing as a next highest degree. Luckily, there is a way to avoid the need for a next highest degree under the approach of increasing discernment: the world ordering $\preccurlyeq^{+}$, defined in (11), simulates the passing down of orderings from higher degrees to lower degrees in a way compatible with a dense ordering of degrees.

$w_{1} \preccurlyeq_{g^{c}, w, d}^{+} w_{2}$ iff $w_{1} \prec_{g^{c}, w, d}^{+} w_{2}$ or $w_{1} \approx_{g^{c}, w, d}^{+} w_{2}$, where:

$$
\begin{aligned}
& \text { a. } w_{1} \prec_{g^{c}, w, d}^{+} w_{2} \text { iff } \exists d^{\prime} \geq d\left[w_{1} \prec_{g^{c}\left(w, d^{\prime}\right)} w_{2} \wedge \forall d^{\prime \prime}>d^{\prime}\left[w_{1} \preccurlyeq g_{g^{c}\left(w, d^{\prime \prime}\right)} w_{2}\right]\right] \\
& \text { b. } w_{1} \approx_{g^{c}, w, d}^{+} w_{1} \text { iff } \forall d^{\prime} \geq d\left[w_{1} \approx_{g^{c}\left(w, d^{\prime}\right)} w_{2}\right]
\end{aligned}
$$

Graphics illustrating the definitions of $\approx^{+}$and $\prec^{+}$can be seen in Figure 1 . The important thing to note is that due to the definition of $\preccurlyeq^{+}$, both strict ordering $\left(\prec^{+}\right)$and incomparability of worlds are passed down from higher degrees to lower degrees, while equivalence $\left(\approx^{+}\right)$is not. Note the similarity between this approach and Katz et al.'s (2012): orderings at lower degrees can only serve as tie-breakers for orderings at higher degrees. 
A conservative theory of gradable modality

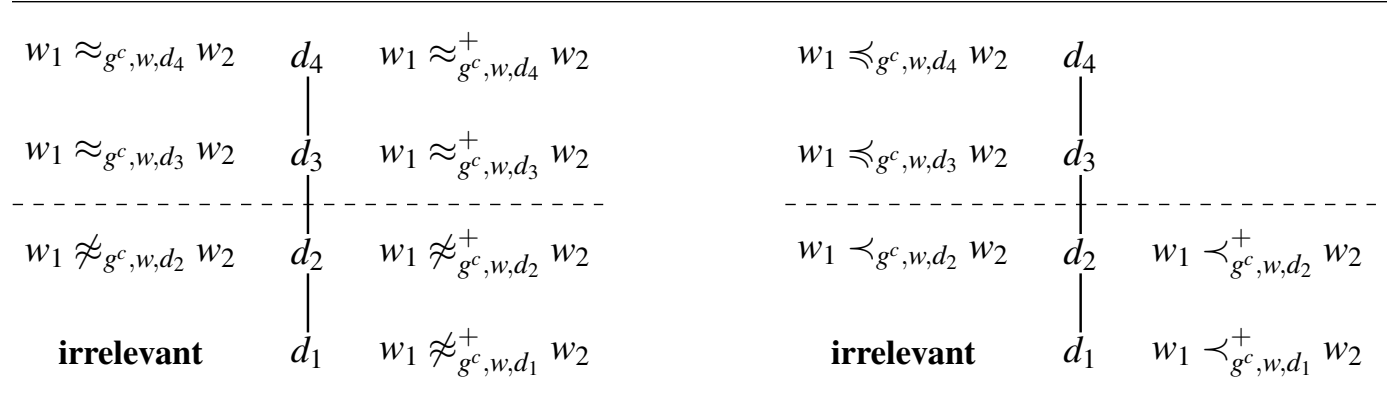

Figure 1 Graphics illustrating definitions of $\approx^{+}$(left) and $\prec^{+}$(right).

For example, let's assume $D$ has just two degrees, $d_{1}$ and $d_{2}$, with $d_{1}<d_{2}$. Furthermore, assume that $g^{c}\left(w, d_{2}\right)=\left\{p_{1}, p_{2}\right\}$, while $g^{c}\left(w, d_{1}\right)=\{q\}$, with $p_{1}, p_{2}$, and $q$ all being logically independent. It might be that we are going out to dinner, and $p_{1}$ is true in all worlds in which I wear a shirt to dinner, $p_{2}$ is true in all worlds in which I wear shoes to dinner, and $q$ is true in all worlds in which I show up to dinner on time. Thus, in the case of this example, wearing the right attire to dinner is of higher priority than showing up on time.

Diagrams of $\preccurlyeq_{g^{c}, w, d_{2}}^{+}$and $\preccurlyeq_{g^{c}, w, d_{1}}^{+}$can be seen in Figure 2. In order to illustrate $\preccurlyeq^{+}$more easily, orderings are shown in terms of equivalence classes of worlds: each proposition in each diagram is a set of worlds that are equivalent to each other in the ordering at hand. Notice that the set of $\preccurlyeq_{g^{c}, w, d_{1}}^{+}$-ideal worlds $\left(p_{1} \cap p_{2} \cap q\right)$ is a proper subset of the set of $\preccurlyeq_{g^{c}, w, d_{2}}^{+}$-ideal worlds $\left(p_{1} \cap p_{2}\right)$. In fact, for the same reason that it is necessarily the case that $\operatorname{BEST}\left(\preccurlyeq A_{1} * A_{2}, F_{w}^{c}\right) \subseteq \operatorname{BEST}\left(\preccurlyeq A_{1}, F_{w}^{c}\right)$ in Katz et al.'s (2012) formalism, by the definition of $\preccurlyeq^{+}$it will always be the case that $\operatorname{BEST}\left(\preccurlyeq_{g^{c}, w, d_{1}}^{+}, F_{w}^{c}\right) \subseteq \operatorname{BEST}\left(\preccurlyeq_{g^{c}, w, d_{2}}^{+}, F_{w}^{c}\right)$ when $d_{1}<d_{2}$. Thus, associating with $d$ the set of worlds BEST $\left(\preccurlyeq_{g^{c}, w, d}^{+}, F_{w}^{c}\right)$ leads to the desired result.

With this in mind, «important $\rrbracket^{c}$ can now be defined following the template in (10), but with one small change: instead of finding the maximum degree such that $p$ holds in all worlds associated with that degree, I will define important as returning the supremum (least upper bound) of that set:

$$
\llbracket \text { important } \rrbracket^{c}=\lambda p \lambda w \cdot \sup \left(\left\{d \mid \forall w^{\prime} \in \operatorname{BEST}\left(\preccurlyeq_{g^{c}, w, d}^{+}, F_{w}^{c}\right)\left[p\left(w^{\prime}\right)\right]\right\}\right)
$$

The reason for this change is that it is possible, given the definition of $\preccurlyeq^{+}$, for the set $\left\{d \mid \forall w^{\prime} \in \operatorname{BEST}\left(\preccurlyeq_{g^{c}, w, d}^{+}, F_{w}^{c}\right)\left[p\left(w^{\prime}\right)\right]\right\}$ to lack a well-defined maximum, e.g. if it is equal to $\left\{d \mid d<d_{\alpha}\right\}$ for some degree $d_{\alpha}$. 
Pasternak
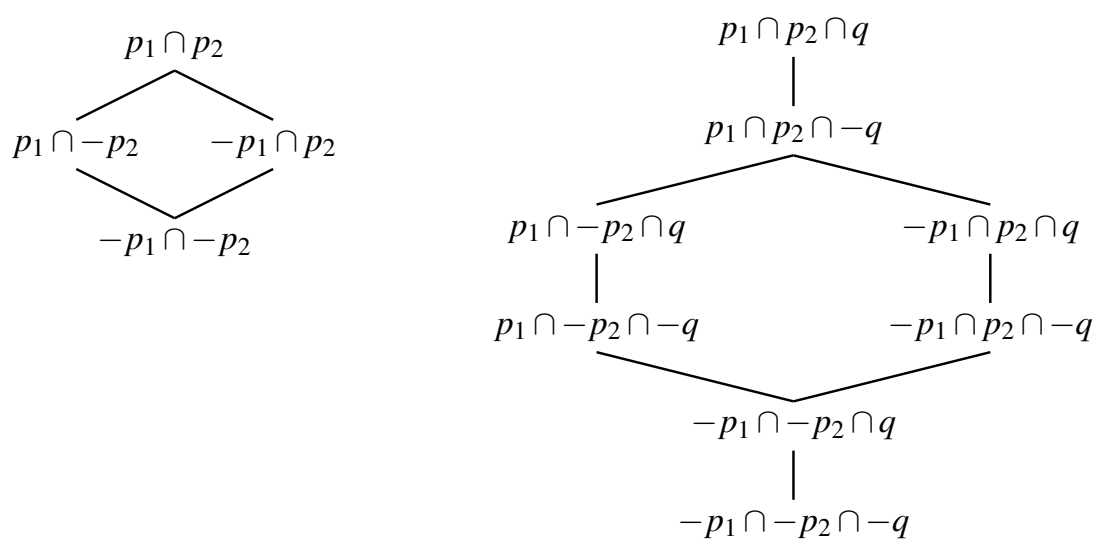

Figure 2 Diagrams of $\preccurlyeq_{g^{c}, w, d_{2}}^{+}$(left) and $\preccurlyeq_{g^{c}, w, d_{1}}^{+}$(right) from the toy example. More ideal sets of worlds are toward the top.

\subsection{Comparing across ordering sources}

With the system now in place, let us once again look at (6). Since this example involves modal evaluation at different times, an additional time argument must be added to the ordering source and modal base. Let $m$ be Monday and $n$ now, and as before, let $p, q$, and $r$ be true in worlds in which Pauline, Quinn, and Rhonda, respectively, are rescued on Saturday. In this case, the sentence in (6) will have the denotation in (13).

$$
\begin{gathered}
\lambda w \cdot \sup \left(\left\{d \mid \forall w^{\prime} \in \operatorname{BEST}\left(\preccurlyeq_{g^{c}, w, m, d}^{+}, F_{w, m}^{c}\right)\left[q\left(w^{\prime}\right)\right]\right\}\right) \geq \\
\sup \left(\left\{d \mid \forall w^{\prime} \in \operatorname{BEST}\left(\preccurlyeq_{g^{c}, w, n, d}^{+}, F_{w, n}^{c}\right)\left[q\left(w^{\prime}\right)\right]\right\}\right)
\end{gathered}
$$

Next, we supply the context from before and see if the truth conditions work out. Let $d_{1}, d_{2}$, and $d_{3}$ be degrees in $D$ such that $d_{1}<d_{2}<d_{3}$. On Monday, Pauline is top priority, followed by Quinn, then Rhonda, so let $g^{c}\left(w, m, d_{3}\right)=\{p\}$, $g^{c}\left(w, m, d_{2}\right)=\{q\}$, and $g^{c}\left(w, m, d_{1}\right)=\{r\}$. The ranking today is reversed, so $g^{c}\left(w, n, d_{3}\right)=\{r\}, g^{c}\left(w, n, d_{2}\right)=\{q\}$, and $g^{c}\left(w, n, d_{1}\right)=\{p\}$. For all degrees $d \notin$ $\left\{d_{1}, d_{2}, d_{3}\right\}, g^{c}(w, m, d)=g^{c}(w, n, d)=\emptyset$. Note that while I have simply switched the places of $p$ and $r$ in the transition from Monday to now, nothing in this theory requires that this be the case. After all, if the CIA switches the rankings of Pauline and Rhonda as before, but at the same time becomes much more laissez-faire, all three propositions could become lower-priority than what they were before, thereby rendering (6) false.

As stated in the scenario, Mary's team is capable of saving all three hostages. Therefore, $p \cap q \cap r \cap F_{w, m}^{c} \neq \emptyset$, and likewise for $F_{w, n}^{c}$. (There is no need to stipulate 


\begin{tabular}{|c|c|c|}
\hline Condition on $d$ & $\operatorname{BEST}\left(\preccurlyeq_{g^{c}, w, m, d}^{+}, F_{w, m}^{c}\right)$ & $\operatorname{BEST}\left(\preccurlyeq_{g^{c}, w, n, d}^{+}, F_{w, n}^{c}\right)$ \\
\hline \hline$d>d_{3}$ & $F_{w, m}^{c}$ & $F_{w, n}^{c}$ \\
\hline$d_{2}<d \leq d_{3}$ & $p \cap F_{w, m}^{c}$ & $r \cap F_{w, n}^{c}$ \\
\hline$d_{1}<d \leq d_{2}$ & $p \cap q \cap F_{w, m}^{c}$ & $q \cap r \cap F_{w, n}^{c}$ \\
\hline$d \leq d_{1}$ & $p \cap q \cap r \cap F_{w, m}^{c}$ & $p \cap q \cap r \cap F_{w, n}^{c}$ \\
\hline
\end{tabular}

Table 1 The sets of ideal worlds for all $d \in D$, on both Monday and today.

that $F_{w, m}^{c}=F_{w, n}^{c}$. Given that the set of worlds that are circumstantially accessible changes over time, this is a welcome consequence of the theory.)

With the context now in place, Table 1 illustrates, for all $d \in D$, what the sets $\operatorname{BEST}\left(\preccurlyeq_{g^{c}, w, m, d}^{+}, F_{w, m}^{c}\right)$ and $\operatorname{BEST}\left(\preccurlyeq_{g^{c}, w, n, d}^{+}, F_{w, n}^{c}\right)$ are. As can be seen in the table, on Monday, the importance of rescuing Quinn on Saturday - and of any proposition that is necessary with regards to rescuing Quinn, but not with regards to rescuing Pauline - is $d_{2}$, as this is the highest degree such that Quinn is rescued on Saturday in all worlds associated with that degree. The same is true of today, modulo replacing Pauline with Rhonda. As a result, (6) is predicted to be true in this scenario.

As for (15), the analysis is essentially the same, except that the difference between the ordering sources is not the time variable, but the contextual parameter. There is one ordering source, $g^{\mathrm{FBI}}$, which includes the FBI's priorities, and another, $g^{\mathrm{CIA}}$, with the CIA's priorities. From there, the comparison takes place just as before.

\subsection{An aside on incompatibles}

P\&R note that the version of their theory articulated in Section 2 runs into problems with comparisons of incompatible propositions like (14):

(14) It is as important to preserve the wetlands as it is to build the new housing (which would drain the wetlands). (Portner \& Rubinstein 2014: 23)

Examples like this are problematic because if preserving the wetlands and building the housing are incompatible, there can be no non-empty set of worlds such that both occur in all members of that set. Hence, if important involves universal quantification over sets of ideal worlds, then it seems that two incompatible propositions cannot be equally important, contrary to (14).

P\&R's response is to abandon universal quantification over all ideal worlds, replacing it with universal quantification over worlds at individual maxima (cf. Kratzer's (1991) definition of "good possibility"). Since a preorder of worlds can have multiple maxima, it is thus possible for all worlds at one maximum to be worlds in which the wetlands are preserved, with another maximum having only worlds 
where the housing is built. However, (14) can just as easily be formulated as a comparison across different sets and/or rankings of priorities, just like (6) and (8). That is, (14) can be thought of as essentially meaning the same thing as (15), which includes priority-specifying in view of phrases à la Kratzer 1977:

(15) It is as important (in view of our environmental priorities) to preserve the wetlands as it is (in view of our financial priorities) to build the new housing.

On this anlaysis, there are simply two ordering sources at play: $g^{\text {env }}$, which includes our environmental priorities, and $g^{\text {fin }}$, which includes our financial priorities. At a given degree $d$, it is possible for all worlds in BEST $\left(\preccurlyeq_{g^{\text {env }}, w, d}, F_{w}^{c}\right)$ to be worlds

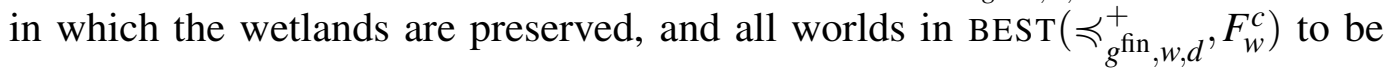
worlds in which the housing is built, even if there are no worlds in $F_{w}^{c}$ in which both take place.

In the analysis adopted in this paper, (14) can thus be treated in a fashion that retains the view of important as involving universal quantification over the set of all ideal worlds. As will be shown in Section 4, such an analysis has benefits not only in terms of intuitive plausibility, but also with respect to capturing entailment relations that are attributable to the dual relationship between universal and existential quantification.

\section{Existential quantification and cross-polarity}

Next, I extend this analysis from universally-quantifying GMAs like important to existentially-quantifying GMAs like permissible. Of particular importance to this extension will be the fact that (16a) and (16b) are mutually entailing:

(16) a. It is more important that you do the dishes than it is that you walk the dog.

b. It is more permissible for you to not walk the dog than it is for you to not do the dishes.

The mutual entailment in (16), represented in a template form in (17), bears a prima facie resemblance to inferences involving antonymous adjectives like tall/short and dirty/clean, as in (18):

(17) $p$ is more important than $q$ iff $-q$ is more permissible than $-p$.
a. $x$ is taller than $y$ iff $y$ is shorter than $x$.
b. $x$ is dirtier than $y$ iff $y$ is cleaner than $x$.

These parallels are further bolstered by the fact that comparing the importance of $p$ with the permissibility of $q$ (or $-q$ ), much like comparing the tallness (or dirtiness) of $x$ with the shortness (or cleanness) of $y$, leads to deviance: 
A conservative theory of gradable modality

(19) a. \# It is more important that you do the dishes than it is permissible for you to not walk the dog. ${ }^{5}$

b. \# Sandra is taller than Ann is short.

c. \# The floor is dirtier than the windows are clean.

Thus, by all appearances, important and permissible are opposite-polarity adjectives like tall/short and dirty/clean, but with the additional caveat that the inference in (17) requires the inclusion of negation where the inferences in (18) do not.

I will treat both of these facts as resulting from the dual nature of universal and existential quantification. The analysis of important in this paper has relied on the plausible assertion that the more demanding one has to be in order to mandate $p$, the less important $p$ is. But there is another, related assertion that is equally plausible: the more demanding one has to be in order to forbid $p$ (or, equivalently, the more demanding that one can be while still allowing $p$ ), the more permissible $p$ is. This intuition is easy to carry over to the apparatus developed so far. Because $\operatorname{BEST}\left(\preccurlyeq_{g^{c}, w, d_{1}}^{+}, F_{w}^{c}\right) \subseteq \operatorname{BEST}\left(\preccurlyeq_{g^{c}, w, d_{2}}^{+}, F_{w}^{c}\right)$ if $d_{1}<d_{2}$, not only does universal quantification get weaker at lower degrees, but existential quantification gets stronger. Therefore, in accordance with our intuition, we can claim in informal terms that $p$ is more permissible than $q$ iff the distance we can go down the scale while retaining ideal worlds in which $p$ holds is greater than the distance we can go down the scale while retaining ideal worlds in which $q$ holds.

On such an analysis, (17) falls out immediately. Because universal and existential quantification are duals, the sets $\left\{d \mid \forall w^{\prime} \in \operatorname{BEST}\left(\preccurlyeq_{g^{c}, w, d}^{+}, F_{w}^{c}\right)\left[p\left(w^{\prime}\right)\right]\right\}$ and $\left\{d \mid \exists w^{\prime} \in\right.$ $\left.\operatorname{BEST}\left(\preccurlyeq_{g^{c}, w, d}^{+}, F_{w}^{c}\right)\left[\neg p\left(w^{\prime}\right)\right]\right\}$ will always serve to partition the set $D$ of modal degrees, with the former constituting some bottom portion of the scale, and the latter being the top portion. Thus, if the degrees at which $p$ holds in all ideal worlds extend farther upward than the degrees at which $q$ holds in all ideal worlds (i.e., $p$ is more important than $q$ ), then the degrees at which $-q$ holds in some ideal world extend farther downward than the degrees at which $-p$ holds in some ideal world (i.e., $-q$ is more permissible than $-p$ ).

The next step is to actually implement this intuition. I follow Kennedy (1997, 2001) in analyzing cross-polarity by switching from individual degrees to extents.

5 One might claim that this deviance is due to the fact that the complement of important is a that clause, while the complement of permissible is a for... to clause. However, as (i) shows, important comparatives with one that clause and one for. . to clause are well-formed. Furthermore, (ii), which only makes use of for ... to clauses, is as bad as (19a).

(i) It is more important for you to do the dishes than it is that you walk the dog.

(ii) \# It is more important for you to do the dishes than it is permissible for you to not walk the dog. 
Extents are sets of degrees with two important properties. First, they are convex: if an extent $\varepsilon$ is such that $d_{1}, d_{3} \in \varepsilon$, and $d_{1}<d_{2}<d_{3}$, then $d_{2} \in \varepsilon$. Second, they are topologically closed: an extent must contain all of its limit points, so that $\left\{d \mid d \leq d_{\alpha}\right\}$ is an extent, while $\left\{d \mid d<d_{\alpha}\right\}$ is not. ${ }^{6}$ Extents are ordered by $\leq_{e}$, where $\varepsilon_{1} \leq_{e} \varepsilon_{2}$ iff $\varepsilon_{1} \subseteq \varepsilon_{2}$.

For our purposes, two types of extents are worth noting: upward extends, which go upward from the bottom of the scale, and downward extents, going from the top of the scale downward. Since going up the scale strengthens universal quantification, while going down the scale strengthens existential quantification, the definition of important in (20) below will always return an upward extent, and that of permissible a downward extent:

(20) If $\operatorname{cl}(\delta)$ is the closure (the smallest closed superset) of $\delta$, then
a. $\llbracket$ important $\rrbracket^{c}=\lambda p \lambda w \cdot \operatorname{cl}\left(\left\{d \mid \forall w^{\prime} \in \operatorname{BEST}\left(\preccurlyeq_{g^{c}, w, d}^{+}, F_{w}^{c}\right)\left[p\left(w^{\prime}\right)\right]\right\}\right)$
b. $\llbracket$ permissible $\rrbracket^{c}=\lambda p \lambda w \cdot \operatorname{cl}\left(\left\{d \mid \exists w^{\prime} \in \operatorname{BEST}\left(\preccurlyeq_{g^{c}, w, d}^{+}, F_{w}^{c}\right)\left[p\left(w^{\prime}\right)\right]\right\}\right)$

Given the definitions in (20), if $p_{\text {dishes }}$ is true in worlds in which you do the dishes, and $p_{\text {dog }}$ is true in worlds in which you walk the dog, the denotations of (16a) and (16b) are (21a) and (21b), respectively:

$$
\begin{aligned}
& \text { a. } \lambda w \cdot \operatorname{cl}\left(\left\{d \mid \forall w^{\prime}\right.\right.\left.\left.\in \operatorname{BEST}\left(\preccurlyeq_{g^{c}, w, d}^{+}, F_{w}^{c}\right)\left[p_{\text {dishes }}\left(w^{\prime}\right)\right]\right\}\right)>_{e} \\
& \operatorname{cl}\left(\left\{d \mid \forall w^{\prime}\right.\right.\left.\left.\in \operatorname{BEST}\left(\preccurlyeq_{g^{c}, w, d}^{+}, F_{w}^{c}\right)\left[p_{\text {dog }}\left(w^{\prime}\right)\right]\right\}\right) \\
& \text { b. } \lambda w \cdot \operatorname{cl}\left(\left\{d \mid \exists w^{\prime} \in \operatorname{BEST}\left(\preccurlyeq_{g^{c}, w, d}^{+}, F_{w}^{c}\right)\left[\neg p_{\operatorname{dog}}\left(w^{\prime}\right)\right]\right\}\right)>_{e} \\
& \operatorname{cl}\left(\left\{d \mid \exists w^{\prime} \in \operatorname{BEST}\left(\preccurlyeq_{g^{c}, w, d}^{+}, F_{w}^{c}\right)\left[\neg p_{\text {dishes }}\left(w^{\prime}\right)\right]\right\}\right)
\end{aligned}
$$

I leave it to the reader to prove to his or her own satisfaction that (21a) and (21b) are equivalent, as desired.

This analysis also explains the ill-formedness of (19a). For Kennedy (1997, 2001), the deviance of (19b-c) is due to the fact that the extents $\varepsilon_{1}$ and $\varepsilon_{2}$ are only comparable by $\leq_{e}$ if $\varepsilon_{1} \subseteq \varepsilon_{2}$ or $\varepsilon_{2} \subseteq \varepsilon_{1}$, so upward extents and downward extents, which do not stand in the subset relation to each other, will be incomparable. The same reasoning applies here: since the importance of a proposition is an upward extent, and the permissibility of a proposition is a downward extent, comparing the importance of one proposition with the permissibility of another will be ill-formed.

6 Of course, this presupposes that a topology on $D$ has been defined. I assume a topology on $D$ that is homeomorphic to the normal topology on some convex subset of the real or rational numbers. In addition, note that topological closure is distinct from the notion of closure as discussed in work on scale structure (see, e.g., Kennedy \& McNally 1999, 2005; Rotstein \& Winter 2004), since a set can extend infinitely downward, infinitely upward, or even both, while still containing all its limit points. Finally, Kennedy (2001) states that extents must be non-empty; here I remain agnostic. 
A conservative theory of gradable modality

\section{Positive form and entailments with auxiliaries}

Finally, let us look at the entailments discussed in the introduction, where we saw that must $p$ asymmetrically entails it is important that $p$, which in turn asymmetrically entails should $p$. In analyzing these entailments, I will adopt von Fintel \& Iatridou's (2008) previously discussed theory of weak versus strong necessity modals. Recall that von Fintel \& Iatridou (2008) posit the existence of two ranked ordering sources, $g_{1}^{c}$ and $g_{2}^{c}$, with must quantifying over BEST $\left(\preccurlyeq g_{1}^{c}(w), F_{w}^{c}\right)$, the set of ideal worlds with respect to $\preccurlyeq g_{1}^{c}(w)$. In the case of should, these ideal worlds are then passed down to $\preccurlyeq g_{2}^{c}(w)$, resulting in quantification over $\operatorname{BEST}\left(\preccurlyeq g_{2}^{c}(w), \operatorname{BEST}\left(\preccurlyeq g_{1}^{c}(w), F_{w}^{c}\right)\right)$. Since the latter set is a subset of the former, universal quantification over the latter is weaker than universal quantification over the former, so the right entailments are derived.

In extending this account to the degree-relative approach discussed in the previous two sections, I will limit the inclusion of degrees to $g_{2}^{c}$, so that $g_{1}^{c}$ will operate in a straightforwardly Kratzerian manner. In other words, degrees will be involved in the denotations of important and should, but not in the denotation of must. This exclusion of degrees from $g_{1}^{c}$ is done out of a desire for brevity and technical simplicity; I leave it to future work to determine whether degrees should actually be included there as well.

With this in mind, we can treat must exactly like von Fintel \& Iatridou (2008) do, defining it as universal quantification over worlds in $\operatorname{BEST}\left(\preccurlyeq g_{1}^{c}(w), F_{w}^{c}\right)$, which I abbreviate as $\operatorname{DOM}^{c}(w)$ for ease of reading.

$$
\llbracket m u s t \rrbracket^{c}=\lambda p \lambda w . \forall w^{\prime} \in \operatorname{DOM}^{c}(w)\left[p\left(w^{\prime}\right)\right]
$$

Turning now to important, in order to allow for the existence of multiple ordering sources, we must again update our definition by tweaking the one in (20a), replacing $F_{w}^{c}$ with $\operatorname{DOM}^{c}(w)$. This way, only worlds that are ideal with respect to $\preccurlyeq g_{1}^{c}(w)$ are under consideration:

$$
\llbracket \text { important } \rrbracket^{c}=\lambda p \lambda w \cdot \operatorname{cl}\left(\left\{d \mid \forall w^{\prime} \in \operatorname{BEST}\left(\preccurlyeq_{g_{2}^{c}, w, d}^{+}, \operatorname{DOM}^{c}(w)\right)\left[p\left(w^{\prime}\right)\right]\right\}\right)
$$

Finally, we are left with should. Presumably, should ought to have the same semantic type as must, rather than important: after taking a proposition and a world of evaluation, it returns a truth value, rather than a degree. With this in mind, I propose the definition of should in (24), which posits that should $p$ is true iff $p$ has at least some importance.

$$
\llbracket \text { should } \rrbracket^{c}=\lambda p \lambda w .\left\{d \mid \forall w^{\prime} \in \operatorname{BEST}\left(\preccurlyeq_{g_{2}^{c}, w, d}^{+}, \operatorname{DOM}^{c}(w)\right)\left[p\left(w^{\prime}\right)\right]\right\} \neq \emptyset
$$

Before discussing how these definitions adequately capture the entailments between must $p$, it is important that $p$, and should $p$, it is necessary to address how 
the positive use of gradable adjectives (e.g., John is tall, in contrast to John is taller than Bill) is handled within a theory in which the denotations of gradable adjectives are measure functions. Kennedy $(1997,2007)$, who adopts such a theory, follows von Stechow (1984) in positing the presence of an unpronounced morpheme POS, which combines with the adjective to make an implicit comparative that returns true iff the extent returned by the measure function is no less than some contextually determined standard extent. If $\varepsilon_{S}$ is this standard extent, the denotation of POS important will be as seen in (25):

$$
\begin{aligned}
& \llbracket \text { POS important } \rrbracket^{c}= \\
& \qquad \lambda p \lambda w \cdot \operatorname{cl}\left(\left\{d \mid \forall w^{\prime} \in \operatorname{BEST}\left(\preccurlyeq_{g_{2}^{c}, w, d}^{+}, \operatorname{DOM}^{c}(w)\right)\left[p\left(w^{\prime}\right)\right]\right\}\right) \geq_{e} \varepsilon_{s}
\end{aligned}
$$

Assuming that the pronoun it in it is POS important that $p$ is semantically idle, the denotation of it is POS important that $p$ will be the denotation in (25) applied to $p$.

From these definitions, the entailment from must $p$ to it is POS important that $p$ comes without stipulation. If $\llbracket m u s t p \rrbracket^{c}$ is true in world $w$, then $p$ holds in all worlds in $\operatorname{DOM}^{c}(w)$. Since for any given degree $d, \operatorname{BEST}\left(\preccurlyeq_{g^{c}, w, d}^{+}, \operatorname{DOM}^{c}(w)\right) \subseteq \operatorname{DOM}^{c}(w), p$ will also hold at all worlds associated with each degree, so that $\llbracket$ important $\rrbracket^{c}(p)(w)=$ $D$, the set of all modal degrees. No matter what $\varepsilon_{s}$ is, $D \geq_{e} \varepsilon_{s}$ because $D \supseteq \varepsilon_{s}$, so $\llbracket$ POS important $\rrbracket^{c}(p)(w)$ is guaranteed to be true.

Furthermore, if $\varepsilon_{s} \neq \emptyset$ (an assumption that can be taken for granted, or else every proposition would be vacuously important), the entailment from it is POS important that $p$ to should $p$ follows as well. Using $\delta$ as shorthand for the set $\left\{d \mid \forall w^{\prime} \in \operatorname{BEST}\left(\preccurlyeq_{g_{2}^{c}}^{+}, w, d, \operatorname{DOM}^{c}(w)\right)\left[p\left(w^{\prime}\right)\right]\right\}$, the former asserts that $\operatorname{cl}(\delta) \geq_{e} \varepsilon_{s}$, which, by the definition of $\geq_{e}$, is equivalent to $\operatorname{cl}(\delta) \supseteq \varepsilon_{s}$. Since $\varepsilon_{s} \neq \emptyset$, we know that it is also the case that $\operatorname{cl}(\delta) \neq \emptyset$. In addition, note that the empty set is topologically closed, since it vacuously contains all its limit points. Thus, $\operatorname{cl}(\emptyset)=\emptyset$, so if $\operatorname{cl}(\delta) \neq \emptyset$, then $\delta \neq \emptyset$, which is exactly what should $p$ asserts.

\section{Conclusion}

In this paper, I have shown that previous conservative theories of gradable modality cannot properly handle cases of comparison across ordering sources. I resolved this problem by including modal degrees in the ontology, while still retaining the premise-based world ordering and quantification that lies at the heart of conservative theories. I then extended this theory to account for existentially-quantifying GMAs, as well as inferences between GMAs and modal auxiliaries. The result was a theory that remained faithful to traditional approaches to the semantics of modality, but without stipulating a fundamental difference between modal and non-modal gradability. 
A conservative theory of gradable modality

\section{References}

Beck, Sigrid. 2011. Comparison constructions. In Klaus von Heusinger, Claudia Maienborn \& Paul Portner (eds.), Semantics: An International Handbook of Natural Language Meaning Vol. 2, 1341-1390. Berlin: de Gruyter. doi:10.1515/9783110255072.1341.

von Fintel, Kai \& Sabine Iatridou. 2008. How to say 'ought' in foreign: The composition of weak necessity modals. In Jacqueline Guéron \& Jacqueline Lecarme (eds.), Time and Modality (Studies in Natural Language and Linguistic Theory 75), 115-141. Springer. doi:10.1007/978-1-4020-8354-9_6.

Katz, Graham, Paul Portner \& Aynat Rubinstein. 2012. Ordering combination for modal comparison. In Anca Chereches (ed.), Semantics and Linguistic Theory (SALT) 22, 488-507. doi:10.3765/salt.v0i0.2647.

Kennedy, Christopher. 1997. Projecting the adjective: The syntax and semantics of gradability and comparison. Santa Cruz, CA: University of California Santa Cruz PhD dissertation.

Kennedy, Christopher. 2001. Polar opposition and the ontology of 'degrees'. Linguistics and Philosophy 24(1). 33-70.

Kennedy, Christopher. 2007. Vagueness and grammar: The semantics of relative and absolute gradable adjectives. Linguistics and Philosophy 30(1). 1-45. doi:10.1007/s10988-006-9008-0.

Kennedy, Christopher \& Louise McNally. 1999. From event structure to scale structure: Degree modification in deverbal adjectives. In T. Matthews \& D. Strolovitch (eds.), Semantics and Linguistic Theory (SALT) 9, 163-180. doi:10.3765/salt.v0i0.2820.

Kennedy, Christopher \& Louise McNally. 2005. Scale structure, degree modification, and the semantics of gradable predicates. Language 81(2). 345-381. doi:10.1353/lan.2005.0071.

Klecha, Peter. 2014. Bridging the divide: Scalarity and modality. Chicago, IL: University of Chicago $\mathrm{PhD}$ dissertation.

Kratzer, Angelika. 1977. What 'must' and 'can' must and can mean. Linguistics and Philosophy 1(3). 337-355. doi:10.1093/acprof:oso/9780199234684.003.0001.

Kratzer, Angelika. 1981. The notional category of modality. In H.J. Eikmeyer \& H. Rieser (eds.), Words, Worlds, and Contexts: New Approaches in Word Semantics, 38-74. Berlin: de Gruyter. doi:10.1515/9783110842524-004.

Kratzer, Angelika. 1991. Modality. In Arnim von Stechow \& Dieter Wunderlich (eds.), Semantik/Semantics: An International Handbook of Contemporary Research, 639-650. Berlin: de Gruyter.

Kratzer, Angelika. 2012. Modals and Conditionals: New and Revised Perspectives. Oxford: Oxford University Press. 
Lassiter, Daniel. 2011a. Measurement and modality: The scalar basis of modal semantics. New York, NY: New York University PhD dissertation.

Lassiter, Daniel. 2011b. Nouwen's puzzle and a scalar semantics for obligation, needs, and desires. In Neil Ashton, Anca Chereches \& David Lutz (eds.), Semantics and Linguistic Theory (SALT) 21, 694-711. doi:http://dx.doi.org/10.3765/salt.v21i0.2608.

Lewis, David. 1973. Counterfactuals. Malden, MA: Blackwell.

Lewis, David. 1981. Ordering semantics and premise semantics for counterfactuals. Journal of Philosophical Logic 10(2). 217-234. doi:10.1007/bf00248850.

Portner, Paul \& Aynat Rubinstein. 2014. Extreme and non-extreme deontic modals. To appear in N. Charlow \& M. Chrisman (eds.), Deontic Modals, Oxford University Press (Pre-final version available at http://semanticsarchive.net/Archive/ TUxZDIyY/).

Rotstein, Carmen \& Yoad Winter. 2004. Total adjectives vs. partial adjectives: Scale structure and higher-order modifiers. Natural Language Semantics 12(3). 259-288. doi:10.1023/b:nals.0000034517.56898.9a.

von Stechow, Arnim. 1984. Comparing semantic theories of comparison. Journal of Semantics 3(1). 1-77. doi:10.1093/jos/3.1-2.1.

Villalta, Elisabeth. 2008. Mood and gradability: an investigation of the subjunctive mood in Spanish. Linguistics and Philosophy 31(4). 467-522. doi:10.1007/s10988-008-9046-x.

Robert Pasternak

Department of Linguistics

Stony Brook University

Stony Brook, NY 11794-4376

robert.pasternak@stonybrook.edu 